\title{
BMJ Open Group education for adolescents with type 1 diabetes during transition from paediatric to adult care: study protocol for a multisite, randomised controlled, superiority trial (GET-IT-T1D)
}

\author{
Elise Mok, ${ }^{1}$ Melanie Henderson, ${ }^{2}$ Kaberi Dasgupta, ${ }^{1}$ Elham Rahme, ${ }^{1}$ \\ Mohammad Hajizadeh, ${ }^{3}$ Lorraine Bell, ${ }^{4}$ Melinda Prevost, ${ }^{5}$ Jennifer Frei, ${ }^{1}$ \\ Meranda Nakhla ${ }^{5}$
}

To cite: Mok E, Henderson M, Dasgupta K, et al. Group education for adolescents with type 1 diabetes during transition from paediatric to adult care: study protocol for a multisite, randomised controlled, superiority trial (GET-IT-T1D). BMJ Open 2019;9:e033806. doi:10.1136/ bmjopen-2019-033806

- Prepublication history and additional material for this paper are available online. To view these files, please visit the journal online (http://dx.doi org/10.1136/bmjopen-2019033806).

Received 22 August 2019 Revised 11 September 2019 Accepted 12 September 2019

Check for updates

(C) Author(s) (or their employer(s)) 2019. Re-use permitted under CC BY-NC. No commercial re-use. See rights and permissions. Published by BMJ.

For numbered affiliations see end of article.

Correspondence to Dr Meranda Nakhla; meranda.nakhla@mcgill.ca

\section{ABSTRACT}

Introduction Transition from paediatric to adult care is challenging for adolescents and emerging adults (ages 18 to 30 years) with type 1 diabetes (T1D). This transition is characterised by a deterioration in glycaemic control (haemoglobin A1c (HbA1c)), decreased clinical attendance, poor self-management and increased acute T1D-related complications. However, evidence to guide delivery of transition care is lacking. Given the effectiveness of group education in adult diabetes glycaemic control and improvements in qualitative measures in paediatric diabetes, group education is a potentially feasible and cost-effective alternative for the delivery of transition care. In emerging adults with T1D, we aim to assess the effectiveness of group education visits compared with usual care on $\mathrm{HbA1c}$, T1D-related complications, psychosocial measures and cost-effectiveness after the transfer to adult care.

Methods and analysis In a multisite, assessor-blinded, randomised, two-arm, parallel-group, superiority trial, 212 adolescents with T1D (ages 17 years) are randomised to 12 months group education versus usual T1D care before transfer to adult care. Visits in the active arm consist of group education sessions followed by usual T1D care visits every 3 months. Primary outcome is change in $\mathrm{HbA} 1 \mathrm{c}$ measured at 24 months. Secondary outcomes are delays in establishing adult diabetes care, T1D-related hospitalisations and emergency department visits, severe hypoglycaemia, stigma, self-efficacy, diabetes knowledge, transition readiness, diabetes distress, quality of life and cost-effectiveness at 12 and 24 months follow-up. Analysis will be by intention-to-treat. Change in $\mathrm{HbA1C}$ will be calculated and compared between arms using differences $(95 \% \mathrm{Cl})$, along with cost-effectiveness analysis. A similar approach will be conducted to examine between-arm differences in secondary outcomes. Ethics and dissemination The study was approved by McGill University Health Centre Research Ethics Board (GET-IT/MP-37-2019-4434, version 'Final 1.0 from November 2018). Study results will be disseminated through peer-reviewed publications.

Trial registration number NCT03703440.
Strengths and limitations of this study

- This is the first randomised controlled trial to evaluate group education visits in adolescents with type 1 diabetes transitioning to adult care, eliminating selection bias.

- Novel features include: (i) patient-driven group education visits, which are more efficient than individual visits, increasing scalability and addressing emerging adults' identified needs for peer support, (ii) integration into usual diabetes care, leveraging on existing resources and increasing cost-effectiveness and (iii) 24 months follow-up, evaluating sustainability of the intervention.

- The trial is patient-driven, multicentre, assessorblinded, adequately powered and uses objective clinically relevant outcome measures (haemoglobin A1c) and validated questionnaires.

- Limitations include unblinded trial participants and care providers, given the educational nature of the intervention.

\section{INTRODUCTION}

Approximately $15 \%$ of youth in North America have a chronic health condition that requires transition from paediatric to adult healthcare. ${ }^{1}$ Transition care is the "purposeful, planned movement of adolescents with chronic health conditions from child-centred to adult-oriented healthcare systems' ${ }^{2}$ While transfer to adult care is an event, transition care is an active process that should begin in adolescence. ${ }^{3}$ The goal of transition care is to provide healthcare that is coordinated, uninterrupted, developmentally-appropriate and to develop decision-making and self-care skills. ${ }^{2}$ More than half of adolescents with chronic health conditions report inadequate support and services during transition to adult 
care. ${ }^{4-6}$ Complicating transition care are changes unique to emerging adulthood (ages 18 to 30 years), ${ }^{7}$ including deterioration in glycaemic control (haemoglobin A1c (HbAlc) $),{ }^{8-11}$ decreased adherence to self-care management $^{1213}$ and clinical attendance, ${ }^{1415}$ increased risk of psychiatric disorders ${ }^{16-18}$ and involvement in risk-taking behaviours. ${ }^{19} 20$ These combined challenges result in an increased risk of inadequate medical follow-up, poor selfmanagement, emergency department (ED) visits and hospitalisations. $^{21}$

Type 1 diabetes (T1D) is one of the most common chronic diseases of childhood with significant morbidity and mortality. ${ }^{22}{ }^{23}$ Prevention of complications is vital for patient health. Randomised trials have demonstrated the importance of optimising glycaemic control in preventing chronic complications. ${ }^{24}$ Prevention of shorter term complications are equally critical in T1D management; diabetic ketoacidosis (DKA) and hypoglycaemia, remain leading causes of preventable hospitalisations, ED visits and death in T1D. 22 23 25-28 The British Diabetic Association Cohort study determined that mortality in the 20- to 29-year age group is three-fold higher in men and six-fold in women with T1D, compared with the general population. ${ }^{29}$ Acute preventable T1D-related complications (ie, hypoglycaemia, DKA) were the most common cause of death under the age of 30 years. ${ }^{30}$ The containment of healthcare costs is also important. The economic cost of one DKA hospitalisation ranges from US $\$ 4125$ to US\$11 196, in addition to familial and societal costs with missed work and school days. ${ }^{28}$

To reduce complications and costs, medical care of adolescents and emerging adults with T1D requires regular access to specialised healthcare services. $^{31}$ However, during transfer to adult care, emerging adults are at risk of dropping out of medical care, only to resurface in the healthcare system with complications. ${ }^{32} 33 \mathrm{~A}$ lack of transition care services was found in paediatric diabetes centres across Quebec, Canada. ${ }^{34}$ Adult diabetes care providers perceive an absence of transition care preparation as a major barrier to successful transition. ${ }^{35}$ Although there is an established framework to guide transition care, there is limited evidence to inform delivery of transition care interventions. ${ }^{3637}$ A Cochrane review identified only four randomised controlled trials (RCTs) evaluating transition care interventions. ${ }^{38}$ These were limited by small sample size, selection bias and short follow-up. The review concluded that there was a need for a stronger evidence-base to inform the development of transition care interventions. ${ }^{38}$ In emerging adults with T1D, only few observational studies have addressed this problem. ${ }^{39}$ These studies focused on transition care coordinators ${ }^{40}$ and specialised young adult clinics. ${ }^{51-43}$ Although, these are promising interventions, they are restricted by costs, need for additional resources and limited scalability. Furthermore, these observational studies were limited by small sample size, selection bias, lack of appropriate comparison group and lack of adjustment for confounding factors. ${ }^{44}$ As well data on $\mathrm{ED}$ visits were not available.
Group education visits are an alternative for delivering transition care. These visits are characterised by a group of patients receiving comprehensive patient education, counselling and peer support in an interactive environment. ${ }^{3645}$ Emerging adults' 'wish lists' for T1D transition care include a need for education and preparation for transition care as well as peer support. ${ }^{44-48}$ Currently, paediatric diabetes centres have interdisciplinary healthcare teams that include diabetes nurse educators and dietitians who provide one-on-one education. Group education visits may be more efficient for providing this education. ${ }^{49}$ Compared with one-on-one appointments, group education visits have the potential to provide more in-depth discussions and education on diabetes-related topics, as well as the opportunity for peer support, with minimal additional resources. ${ }^{36} 5051$ Peer support has been demonstrated to improve adherence to diabetes self-care and reduce diabetes-related stress in adolescents and emerging adults with T1D. ${ }^{52}$ In adults with diabetes, group visits have been shown to improve, or stabilise HbAlc, improve self-care, adherence, diabetes knowledge and quality of life (QOL). ${ }^{45554}$ In a meta-analysis, group medical visits in adults with diabetes, resulted in reductions in HbAlc $(-0.46 \%, 95 \%$ CI: $-0.80 \%$ to $-0.31 \%) .{ }^{45}$ In adolescents with $\mathrm{T} 1 \mathrm{D}$, three pilot studies demonstrated the feasibility and acceptability of group visits; however, these studies did not focus on the effect of group education visits on outcomes following transfer to adult care and no RCTs have been conducted. ${ }^{55-57}$

\section{Objectives}

The primary aim of our study is to determine if group education visits for adolescents with T1D, integrated into paediatric care is superior to usual care, as measured by change in HbA1c after transfer to adult care. Secondary aims will be to determine if group education is superior to usual care as measured by differences in ${ }^{1}$ : Delay in care between last paediatric and first adult diabetes care visit, ${ }^{2}$ Diabetes-related ED visits and hospitalisations and severe hypoglycaemic events, ${ }^{3}$ Psychosocial measures, including validated measures of stigma, self-efficacy, QOL and diabetes distress, ${ }^{4}$ Disease knowledge and transition readiness and ${ }^{5}$ Cost-effectiveness.

\section{METHODS AND ANALYSIS Trial design}

GET-IT-T1D is a multisite, assessor-blinded, randomised controlled, parallel group, two-arm, 1:1 allocation ratio, superiority trial, evaluating the efficacy of group education visits (active arm) compared with usual care (control arm) in adolescents with T1D transferring from paediatric to adult care. Participants will be randomised to 12 months of group education visits versus usual care in the year before transfer. Assessments will be performed at 0 months (baseline; before transfer), 12 months (end of intervention; at transfer) and 24 months (12 months after 
$\underline{\text { Schedule of enrolment, interventions, and assessments }}$

\begin{tabular}{|c|c|c|c|c|c|c|}
\hline \multirow[b]{2}{*}{ Time Point } & \multirow{2}{*}{$\begin{array}{l}\text { Enrolment/ } \\
\text { Allocation } \\
-3 \text { months }\end{array}$} & \multicolumn{3}{|c|}{ Intervention Period } & \multirow{2}{*}{$\begin{array}{l}\text { End of } \\
\text { Intervention }\end{array}$} & \multirow{2}{*}{$\begin{array}{l}\begin{array}{l}\text { Post- } \\
\text { Intervention } \\
\text { Follow-up }\end{array} \\
24 \text { months } \\
\end{array}$} \\
\hline & & 0 & Every 3 months & 12 months & & \\
\hline \multicolumn{7}{|l|}{ ENROLMENT } \\
\hline Eligibility screen & $\mathrm{x}$ & & & & & \\
\hline Informed consent & $\mathrm{X}$ & & & & & \\
\hline Allocation & $\mathrm{X}$ & & & & & \\
\hline \multicolumn{7}{|l|}{ INTERVENTIONS } \\
\hline Active arm & & $\bullet$ & & & & \\
\hline Control arm & & $\bullet$ & & $\rightarrow$ & & \\
\hline \multicolumn{7}{|l|}{ ASSESSMENTS } \\
\hline HbAlc (\%) & & $\mathrm{x}$ & & & $\mathrm{x}$ & $\mathrm{x}$ \\
\hline $\begin{array}{l}\text { Delay in establishing adult } \\
\text { T1D care }\end{array}$ & & $\mathrm{X}$ & & & $\mathrm{X}$ & $\mathrm{X}$ \\
\hline $\begin{array}{l}\text { T1D-related } \\
\text { hospitalizations }\end{array}$ & & $x$ & & & $\mathrm{x}$ & $\mathrm{X}$ \\
\hline $\begin{array}{l}\text { T1D-related emergency } \\
\text { department visits }\end{array}$ & & $\mathrm{x}$ & & & $\mathrm{x}$ & $\mathrm{X}$ \\
\hline Severe hypoglycemia & & $\mathrm{X}$ & & & $\mathrm{X}$ & $\mathrm{x}$ \\
\hline Stigma (BDA) 3 questions & & $\mathrm{x}$ & & & $\mathrm{X}$ & $\mathrm{X}$ \\
\hline Self-efficacy (SEDM) & & $x$ & & & $\mathrm{x}$ & $\mathrm{x}$ \\
\hline $\begin{array}{l}\text { Diabetes knowledge } \\
\text { (AJD-DKS) }\end{array}$ & & $\mathrm{X}$ & & & $\mathrm{x}$ & $\mathrm{x}$ \\
\hline $\begin{array}{l}\text { Transition readiness } \\
\text { (Am I ON TRAC?) }\end{array}$ & & $\mathrm{X}$ & & & $\mathrm{x}$ & \\
\hline $\begin{array}{l}\text { Diabetes distress } \\
\text { (T1-DDS) }\end{array}$ & & $x$ & & & $\mathrm{x}$ & $\mathrm{x}$ \\
\hline QOL (PedsQL) & & $\mathrm{x}$ & & & $\mathrm{X}$ & $\mathrm{X}$ \\
\hline
\end{tabular}

Figure 1 Participant timeline. AJD-DKS, L'Aide aux Jeunes Diabétiques Diabetes Knowledge and Skills questionnaire; Am I ON TRAC?, Am I ON TRAC? For Adult Care questionnaire; BDA, Barriers to Diabetes Adherence in Adolescence questionnaire stigma subscale; $\mathrm{HbA1c}$, haemoglobin A1c; PedsQL, Paediatric Quality of Life Inventory Generic Core Scale and Diabetes Module; QOL, quality of life; SEDM, Self-Efficacy for Diabetes SelfManagement Measure; T1D, type 1 diabetes; T1-DDS, Diabetes Distress Scale for Adults with T1D.

end of intervention; after transfer). Figure 1 presents the participant timeline.

\section{Study setting and eligibility criteria}

Participants are adolescents 16 to 17 years of age, diagnosed with T1D, receiving care at a paediatric diabetes clinic at two academic hospitals in Montreal, Canada. Exclusion criteria are severe neurocognitive disabilities, or non-fluent in English or French. Study sites are the Montreal Children's Hospital, McGill University Health Centre (MUHC) and the Centre Hospitalier Universitaire Sainte-Justine (CHUSJ). A certified diabetes educator (nurse, dietitian, social worker) will facilitate group education visits.

\section{Interventions}

Control arm participants will receive usual care, consisting of routine clinic visits with their diabetes care physician every 3 months for 12 months and an education session with a nurse providing written information before formal transfer. Active arm participants will be asked to attend at least three group education sessions plus usual care, every 3 months for 12 months. Sessions will coincide with

\begin{tabular}{ll}
\hline Table 1 & Discussion topics in group education visits \\
\hline Session & Topic/theme \\
\hline 1 & Alcohol, drugs and smoking \\
& Interpersonal relationships \\
& Driver's permit and driving \\
& Travel \\
& Food \\
Insulin \\
Hypoglycaemia \\
Hyperglycaemia \\
Physical activity \\
School/work \\
Stress \\
Parents \\
Moving out \\
Special events \\
Adult diabetes care \\
Medical insurance and tax credit \\
Complications of diabetes \\
Diabetes technology \\
Free topic(s) based on participant questions
\end{tabular}

routine clinic visits. Three to eight patients will be scheduled per group. Participants will remain with the same group throughout the 12-month intervention. However, a given trial participant may change groups, on participant request. Further, participants who miss the scheduled group education visit could attend the next available group visit. Sessions will commence with an ice-breaker activity (participant introductions) and then move to patient-driven, facilitator-mediated discussions. Sessions will last approximately 1 hour. To begin the participantdriven discussion, group members will be invited to submit written questions anonymously. Facilitators will then rotate reading and answering questions. Participants will be encouraged by the facilitator to ask questions. The group session content will be guided by group participant needs; however, the facilitator will actively promote discussion on transition-related topics and preparation skills, including, self-management, managing diabetes in school/employment and away from home and navigating the adult healthcare system. Table 1 outlines the discussion topics. The group discussion will end with participants setting goals for their next visit. The intervention was informed by the American Diabetes Association's position statement on transition care ${ }^{58}$ the transition care literature, ${ }^{41}$ as well as the clinical and transition care experience of research team members. Strategies to improve adherence include integrating group education into routine clinic visits, requiring no additional study visits or travel/time commitment and gift card incentives for post-intervention and follow-up assessments. 
Participants will also be contacted by telephone, text or email to complete follow-up assessments. Adherence will be monitored by documenting attendance to group education visits.

\section{Recruitment}

Participants will be recruited over 24 months. Recruitment strategies include verbal invitation by a research coordinator (in person or by telephone) following introduction by clinical staff (receptionist, nurse, physician), study information letter and poster advertisements available in clinical waiting areas. Participants will be offered $50 \%$ chance of receiving group education visits at no cost and gift card incentives for participation in assessments. We will obtain ethics approval from the Commission d'Accès à l'Information to merge patient clinical and questionnaire data with administrative data.

\section{Outcomes}

Primary outcome is change in HbAlc (\%) at 24 months from baseline, measured using a capillary blood sample. ${ }^{59}$ HbAlc is measured at 24 months follow-up (12 months after end of intervention) to inform on the sustained effect of our intervention after transfer. Recent findings in the T1D Exchange Registry, a US multicentre registry of individuals with T1D, demonstrated that HbA1c is highest between ages 13 to 25 years and does not stabilise until after age 30 years. ${ }^{10}$ Further, the highest reported HbA1c (9.2\%) was at 19 years of age. Thus, a finding of HbA1c stabilisation with group education would be considered a clinically relevant outcome, given the deterioration in HbAlc after transfer. Change in HbAlc at 12 months follow-up (end of intervention) will inform about the immediate effects of our intervention.

Secondary outcomes (1) Delay in establishing adult diabetes care (at 24 months) derived from the Physician Service Claims Database (RAMQ) and medical record chart. Delay is defined as a delay greater than 6 months between the last paediatric and the first adult diabetes care visit. We chose to examine delays greater than 6 months, since the American Diabetes Association (ADA) recommends diabetes care visits at least every 6 months for individuals aged $>18$ years treated with insulin. ${ }^{58}$ Delay will be expressed as a binary variable and a continuous variable (time in days between the last paediatric and first adult diabetes care visit). (2) T1D-related hospitalisations in the past 12 months (at 0, 12, 24 months), expressed as number of hospitalisations per person-years at risk, and derived from the Hospital Discharge Database (MedEcho) and medical record chart. (3) T1D-related ED visits in the past 12 months (at $0,12,24$ months), expressed as number of ED visits per person-years at risk and derived from the Physician Service Claims Database (RAMQ) and medical record chart. (4) Severe hypoglycaemic events in the past 12 months (at $0,12,24$ months) derived from patient report. (5) Stigma (at 0, 12, 24 months), defined as an affirmative response to at least one of three key items on the Barriers to Diabetes Adherence in Adolescence questionnaire stigma subscale. ${ }^{60}$ (6) Selfefficacy assessed using the Self-Efficacy for Diabetes SelfManagement Measure (at 0, 12, 24 months). Self-efficacy is the belief that one can carry out specific behaviours in specified situations. ${ }^{61}$ In adolescents, self-efficacy is associated with better $\mathrm{HbAlc}$ and adherence to diabetes care. $^{62-64}$ (7) Diabetes knowledge assessed using L'Aide aux Jeunes Diabétiques Diabetes Knowledge and Skills questionnaire (at $0,12,24$ months) ${ }^{65-67}$ (8) Transition readiness measured using the Am I ON TRAC? For Adult Care questionnaire (at 0,12 months) ${ }^{68-70}$ (9) Diabetes distress assessed using the Diabetes Distress Scale for Adults with T1D (at 0,12, 24 months), previously used in adolescents. ${ }^{71} 72$ (10) QOL (health-related and diabetesspecific) assessed using the Paediatric Quality of Life Inventory Generic Core Scale and Diabetes Module (at $0,12,24$ months). (11) Cost-effectiveness of group education visits compared with usual care in terms of incremental cost effectiveness ratios (ICER) for HbA1c, QOL and self-efficacy (at 12, 24 months).

\section{Sample size}

A pilot study testing the effect of group education visits on changes in HbA1 $c^{55}$ demonstrated a stabilisation of HbAlc by the end of the intervention compared with deterioration in $\mathrm{HbAlc}$ in the 9 months leading up to the study by an absolute difference of $0.7 \%$. To be conservative, we aim to detect a $0.5 \%$ absolute difference in HbAlc between intervention and control arms. Based on a SD of $1.2,{ }^{55}$ we require 92 participants per arm to have $80 \%$ power with a 0.05 two-sided alpha using t-test. Allowing a loss to follow-up of up to $15 \%,{ }^{55}$ we require a sample size of 212 participants.

\section{Assignment of interventions \\ Sequence generation}

The allocation sequence will be computer generated by the Unité de recherche clinique appliquée (URCA) using permuted blocks of random sizes with 1:1 allocation ratio. Randomisation will be stratified by site. Group allocation will be ascertained only after enrolment by research assistants.

\section{Allocation concealment mechanism and implementation}

The randomisation schedule will be held at URCA, to ensure that the person who generates the allocation sequence (data manager) is not the person who enrols participants or assigns participants to the interventions (research assistant). To reduce the possibility of study personnel predicting the allocation sequence and enhance allocation concealment, block sizes will be varied.

\section{Blinding}

Because of the nature of the intervention, participants, care providers and investigators cannot be blinded to group allocation. Outcome assessors and data analysts (StatSciences Inc, Montreal, Canada) will be blinded. 
Table 2 Validity and reliability of study questionnaires

\begin{tabular}{|c|c|c|}
\hline Questionnaire & Details & Validity/reliability \\
\hline $\begin{array}{l}\text { Self-efficacy (Self-Efficacy for Diabetes } \\
\text { Self-Management Measure) }\end{array}$ & $\begin{array}{l}\text { Participants will rate their degree of } \\
\text { certainty in their ability to correctly } \\
\text { carry out } 10 \text { diabetes management } \\
\text { tasks on a } 10 \text {-point Likert scale } \\
\text { ranging from 'not sure at all' to } \\
\text { 'completely sure'. }\end{array}$ & $\begin{array}{l}\text { The test-retest intraclass correlation for the } \\
\text { measure was } 0.89 \text {. The Cronbach's alpha } \\
\text { coefficient } 0.90 \text {. }\end{array}$ \\
\hline $\begin{array}{l}\text { Transition readiness (Am I ON TRAC? } \\
\text { For Adult Care questionnaire) }\end{array}$ & $\begin{array}{l}\text { The questionnaire is a } 25 \text {-item } \\
\text { validated scale consisting of: (1) } \\
\text { knowledge scale with a 4-point Likert } \\
\text { scale ranging from 'strongly disagree' } \\
\text { to 'strongly agree' and a (2) behaviour } \\
\text { index with a 5-point ordinal scale, } \\
\text { 'never' to 'always' and asks youth to } \\
\text { report how frequently they engage in } \\
\text { several health-related behaviours }\end{array}$ & Cronbach's alpha coefficient 0.93 \\
\hline $\begin{array}{l}\text { Diabetes distress (Diabetes Distress } \\
\text { Scale for Adults with Type } 1 \text { Diabetes) }\end{array}$ & A validated 28 -item self-report scale & $\begin{array}{l}\text { Alpha coefficient } 0.91 \text {. The test-retest } \\
\text { reliability was } 0.74 \text {. }\end{array}$ \\
\hline $\begin{array}{l}\text { Quality of life (Paediatric Quality of } \\
\text { Life Inventory Generic Core Scale and } \\
\text { Diabetes Module) }\end{array}$ & $\begin{array}{l}\text { A 23-item and 33-item questionnaire, } \\
\text { age-specific for 13- to 18-year-olds } \\
\text { will be used }\end{array}$ & Cronbach's alpha coefficient 0.88 \\
\hline
\end{tabular}

\section{Data collection}

To ensure comparability of HbA1c at 0, 12 and 24 months and between sites, HbAlc will be measured using a nonfasting, capillary blood sample, mail-in test (Bio-Rad Laboratories Ltd, Montreal, Canada). The test is considered one of the most accurate and comprehensive A1c tests available. ${ }^{59}$ At 0 and 12 months, HbAlc will be performed at clinic visits by participants. At 24 months, participants will receive the $\mathrm{HbA1c}$ test with a prepaid envelope for mailing the sample back to Montreal. ${ }^{46}$ All samples will be analysed at CDL Laboratories (Montreal, Canada). Trained laboratory staff performing analysis of HbAlc will be blinded. To promote participant retention and complete follow-up, participants will receive a gift card on receipt of the capillary blood sample at 24 months.

Patient-reported measures will be sent to participants by email through a platform from Research Electronic Data Capture (REDCap). Trained analysts scoring the questionnaires will be blinded. Table 2 details the validity and reliability of the questionnaires. To minimise loss to follow-up at 24 months, we have limited evaluations to an online survey and offering HbAlc testing at home (mail-in kit with prepaid envelope). To further promote participant retention and complete follow-up at 24 months, participants will be contacted through different methods (telephone, text, email).

The administrative data include: (1) Physician Service Claims Database (RAMQ): contains all records for remunerated services provided in outpatient clinics and EDs. Each record contains information on diagnostic code (Ninth Revision of the International Classification of
Disease (ICD-9)), date of service provision, physician speciality and site where service was provided, as well as unit costs for outpatient and inpatient visits. Physician visits and $\mathrm{ED}$ visits will be identified as those having a diagnosis code of hyperglycaemia (ICD-9 code 250), which includes DKA and hyperosmolar hyperglycaemic coma and those with hypoglycaemia (ICD-9 code 251); (2) Hospital Discharge Database (Med-Echo): contains admission data mandatorily collected from Québec hospitals. Information in the database includes the primary and secondary diagnosis coded by ICD-10-CA, dates of admission to and discharge from hospital. Diabetes-related hospitalisations will be identified as those with diagnoses of hyperglycaemia (ICD-10 codes E10.0 to 14.0 and E10.1 to 14.1), which includes DKA and hyperosmolar hyperglycaemic coma and those with diagnoses of hypoglycaemia (E10.63 to 14.63). Unit costs for hospitalisations will be derived from Med-Echo and RAMQ databases, and from an index (All Patient Refined Diagnosis Related Groups) that factors in the relative intensity of resources used during the hospitalisation, provided by the Ministère de la Santé et des Services sociaux. ${ }^{73}$

\section{Baseline data}

We will collect baseline data (eg, sex) from the medical chart by trained assessors. Age of transfer will be determined from the date of transfer (last diabetes visit with a paediatric care physician). Diabetes duration will be determined from the diagnosis date and measured from baseline. Anthropometrics (height, weight) and vital signs are collected as part of routine clinical care. 
Socioeconomic status will be determined using a validated area-based material and social deprivation index developed by Pampalon ( $1=$ most privileged, $5=$ most deprived), ${ }^{74-76}$ previously used in diabetes studies. ${ }^{77} 78$ This index is assigned to census dissemination areas and will be linked to individual's postal codes at baseline.

\section{Data management}

Participants will be identified by a code number. Only the principal investigators and research team will have access to the key to the code linking the participant's name to the study file. Data entry forms and online questionnaires will be entered through a web browser in real time into a secure central database using REDCap, hosted at URCA. Data will be systematically checked for completeness, consistency and plausibility by routine edit checks implemented in the data management system. Queries generated by the system will be checked by the data manager at URCA and transmitted to the research assistants at trial sites for resolution.

\section{Statistical analysis}

Continuous data will be reported as means with SDs (or median and IQR). Categorical data will be reported as proportions. Primary outcome is change in mean HbAlc measured at 24 months and will be compared between trial arms using differences with 95\% CI. A similar approach will be conducted to examine differences in secondary outcomes. Between-arm differences in means will be calculated using Student's t-tests or non-parametric statistics. Categorical variables will be compared between arms using $\mathrm{X}^{2}$ or Fisher's exact test. To account for repeated measures, secondary analysis will be conducted to calculate within (baseline, 12 months, 24 months) and between arm-differences to evaluate changes. The primary analysis will be by intention-to-treat. If adherence is an issue, the intention-to-treat approach may underestimate the magnitude of the effect, but provides unbiased comparisons between groups. For this reason, we will conduct a secondary 'as treated' analysis that will include only participants who attended $\geq 3$ sessions.

\section{Cost-effectiveness}

We will conduct a health economic evaluation, measuring total direct costs and incremental cost-effectiveness of the intervention from the perspective of the health system to inform policy and future scale up of the interventions. All costs related to the interventions will be collected prospectively from the intervention accounts and costs incurred by the healthcare system, respectively: (a) Costs of intervention activities (eg, diabetes educator, patient time); (b) Costs incurred by the healthcare system (ED visits, hospitalisations). ICERs, described as the ratio of the difference in costs to the difference in outcomes between the group education visits (intervention) and compared with usual care (control), will be calculated for outcome measures; HbAlc, QOL and self-efficacy; 95\% CI will be calculated using bootstrap method.
Data monitoring

An independent data monitoring committee was not considered necessary, because patient safety will not be affected by the intervention which focuses on education and peer support. We consider this trial to provide minimal risk. In case of adverse events, patients will be referred to their treating physician at the MUHC, CHUSJ or other healthcare facilities.

\section{Harms}

Adverse events and serious adverse events in the previous 3 months will be documented in a case report form. Study staff will record the severity, onset and end date, action taken, relationship to the intervention and outcome. Due to the nature of the intervention, we do not expect that serious adverse events related to the intervention will occur. We will ask the patient their permission to inform their treating physician, if they have any severe hypoglycaemic events or if the diabetes distress scale indicates distress. Any serious adverse event will be immediately reported to the principal investigator, the Research Ethics Board and entered into the study database.

\section{Auditing}

For monitoring control, and safety, study files and medical charts, may be examined by a person independent from the investigators and mandated by Canadian or international regulatory authorities, such as Health Canada, as well as by representatives of the study sponsor/institution, or the Research Ethics Board. All these individuals and organisations adhere to policies on confidentiality.

Confidentiality and access to data

Only the investigators and the research team from the MUHC and CHUSJ will have access to the study data or personal information about potential and enrolled participants. Personal information will not be shared. Participants' personal study data will be stored using REDCap. Database access will be username and password protected. Information will not be included in the participants' health record. Data analysis will be performed solely using de-identified data. The study data will be stored for 7 years by the principal investigator on site. The principal investigator will have access to the final trial data set.

\section{Biological specimens}

The blood samples will be kept at the MUHC until sending to CDL Laboratories (Montreal, Canada) for analysis for the exclusive objectives of this study and then destroyed after analysis.

\section{Ancillary and post-trial care}

Since serious adverse events have not been reported with similar interventions, ${ }^{45453}$ no provisions for compensation to those who might suffer harm during trial participation will be made. 
Table 3 Roles and responsibilities

Coordinating centre-principal

investigator and study team

Data management team

\begin{tabular}{|c|c|c|}
\hline \multicolumn{3}{|l|}{ Study preparation } \\
\hline Study grant & $\mathrm{X}$ & \\
\hline Study protocol and revisions & $\mathrm{X}$ & \\
\hline Ethics approval and amendments & $X$ & \\
\hline Case report form (CRF) & $\mathrm{X}$ & $X$ \\
\hline Study manual & $X$ & \\
\hline Training and supervision & $\mathrm{X}$ & $\mathrm{X}$ \\
\hline \multicolumn{3}{|l|}{ Study conduct } \\
\hline Recruitment & $\mathrm{X}$ & \\
\hline Informed consent & $\mathrm{X}$ & \\
\hline Randomisation & & $\mathrm{X}$ \\
\hline Allocation & $x$ & \\
\hline Intervention & $\mathrm{X}$ & \\
\hline Accessing participant medical records & $x$ & \\
\hline Assessments & $x$ & \\
\hline Collecting biospecimens & $x$ & \\
\hline Packaging and shipping biospecimens & $\mathrm{x}$ & \\
\hline Analysing biospecimens* & $\mathrm{X}$ & \\
\hline Study documentation & $\mathrm{x}$ & \\
\hline \multicolumn{3}{|l|}{ Data management } \\
\hline Database development (eCRF†) & & $x$ \\
\hline Data entry & $\mathrm{X}$ & \\
\hline Data verification & $\mathrm{X}$ & $\mathrm{X}$ \\
\hline Data archiving & $x$ & \\
\hline Data queries & $x$ & $x$ \\
\hline \multicolumn{3}{|l|}{ Quality control } \\
\hline Trial oversight & $x$ & \\
\hline Assessment and reporting of SAEsł & $x$ & \\
\hline Monitoring & $x$ & $x$ \\
\hline \multicolumn{3}{|l|}{ Data analysis and reporting } \\
\hline Data analysis plan & $\mathrm{X}$ & \\
\hline Data analysis§ & $\mathrm{X}$ & \\
\hline Publication & $\mathrm{X}$ & \\
\hline
\end{tabular}

*Blinded trained laboratory staff (CDL Laboratories).

†eCRF: electronic Case Report Form.

‡SAE: Serious Adverse Event.

$\S$ Blinded data analyst (StatSciences Inc).

\section{Patient and public involvement}

The study uses an integrated knowledge translation approach where researchers and patients actively collaborate to shape all stages of the research process. The patient partner is an emerging adult with T1D and co-investigator on the protocol. She has contributed in study design, protocol development and designing the intervention. She will continue to contribute as a member of the research team by informing study execution, data collection, interpretation and dissemination. In addition to the patient partnership, patient involvement and peer support are key elements in the implementation of the intervention. The intervention is patient-driven and aims at improving transition, thus addressing adolescents' identified needs for transition care preparation. Emerging adults' 'wish lists' for T1D transition care include a need for education and preparation for transition care as well as peer support. ${ }^{447}$ Furthermore group education visits 
are more efficient than individual visits, providing peer support and can be integrated into usual diabetes care, minimising patient burden. Feedback from adolescents with T1D participating in group education sessions at the MUHC reported among the strengths of group sessions, meeting others with T1D and learning the skills that they would need when they leave paediatric care. The choice of outcomes, including glycaemic control and psychosocial measures for example, QOL were also informed by patient priorities.

The patient partner helped inform the recruitment strategies in clinic (eg, gift card incentives), data collection and retention strategies (eg, mail-in finger-prick capillary blood samples and online self-administered questionnaires). Furthermore, patients participating in the group education sessions will help shape the intervention based on their needs and topics of interest to patients. Intervention burden will be assessed by obtaining the patient perspective. Specifically, participants will be asked to report patient time (in hours) as well as travel costs as part of a cost-effectiveness analysis of the intervention. The patient partner will be involved in the interpretation of findings, media relationships and dissemination. We will meet with the patient partner three times over 4 years to review the research protocol, discuss analysis and results as well as dissemination strategies.

\section{Roles and responsibilities}

The roles and responsibilities within the trial are summarised in table 3. An independent data monitoring and safety committee was not involved (see Data monitoring section).

\section{ETHICS AND DISSEMINATION}

Written informed consent will be obtained by trained research assistants (model consent form available as online supplementary file 1). For protocol amendments (eg, changes to eligibility criteria, outcomes or analyses) approval by the Research Ethics Board will be sought. Important protocol modifications will be communicated to investigators, trial participants and the ClinicalTrials. gov registry.

For dissemination, we will reach our primary target audience through our patient partners, policymakers (via the Québec Ministry of Health), open access journal articles, workshops, media releases and presentations at conferences. Furthermore, the principal investigator is a member of Diabetes Canada Clinical Practice Guidelines expert committee and wrote the recommendations section on transition care, as such she is well positioned to disseminate the research findings to a national audience including healthcare administrators and providers as well as patients.

\footnotetext{
Author affiliations

${ }^{1}$ Centre for Outcomes Research \& Evaluation, Research Institute of the McGill University Health Centre, Montreal, Québec, Canada
}

${ }^{2}$ Centre de Recherche du Centre Hospitalier Universitaire Sainte-Justine, Centre Hospitalier Universitaire Sainte-Justine, Montreal, Québec, Canada

${ }^{3}$ School of Health Administration, Dalhousie University, Halifax, Nova Scotia, Canada ${ }^{4}$ Pediatrics, Nephrology, McGill University Health Centre, Montréal, Québec, Canada ${ }^{5}$ Pediatrics, Endocrinology, McGill University Health Centre, Montreal, Québec, Canada

Contributors MN (principal investigator) conceived and designed the study with input from MeH (Pediatric Endocrinologist), KD, ER (Biostatistician), MoH (Health Economist), LB, EM and MP. EM and JF helped with implementation. MN, MeH (Pediatric Endocrinologist), KD, ER, MoH (Health Economist), LB and MP are grant holders. ER provided statistical expertise in clinical trial design and is conducting the primary statistical analysis. EM drafted the initial manuscript. All authors contributed to refinement of the study protocol and approved the final manuscript.

Funding This work was supported by the Canadian Institutes of Health Research (ClHR) grant number PJT 156096.

Competing interests None declared.

Patient consent for publication Not required.

Ethics approval The study was approved by McGill University Health Centre Research Ethics Board (GET-IT/MP-37-2019-4434, version 'Final 1.0 from November 2018).

Provenance and peer review Not commissioned; peer reviewed for ethical and funding approval prior to submission.

Data availability statement Individual participant data supporting the results reported in the published research articles, after de-identification will be available. Other documents that will be available include the study protocol, statistical analysis plan and informed consent form. The data will be available immediately following article publication and ending 5 years after article publication. Data will be shared with researchers who provide a scientifically sound proposal that has been approved by an independent Institutional Review Board and for analyses that addresses the aims specified in the proposal (e.g. for individual participant data meta-analysis). Proposals should be submitted to meranda.nakhla@mcgill.ca. Data requestors will need to sign a data access agreement. Data will be available up to 5 years following article publication at (Link to be included).

Open access This is an open access article distributed in accordance with the Creative Commons Attribution Non Commercial (CC BY-NC 4.0) license, which permits others to distribute, remix, adapt, build upon this work non-commercially, and license their derivative works on different terms, provided the original work is properly cited, appropriate credit is given, any changes made indicated, and the use is non-commercial. See: http://creativecommons.org/licenses/by-nc/4.0/.

\section{REFERENCES}

1 Newacheck PW, Strickland B, Shonkoff JP, et al. An epidemiologic profile of children with special health care needs. Pediatrics 1998; 102:117-23.

2 Blum RW. Transition to adult health care: setting the stage. J Adolesc Health 1995;17:3-5.

3 Cooley WC, Sagerman PJ, American Academy of Pediatrics, et al. Supporting the health care transition from adolescence to adulthood in the medical home. Pediatrics 2011;128:182-200.

4 Garvey KC, Beste M, Luff D, et al. Experiences of health care transition voiced by young adults with type 1 diabetes: a qualitative study. Adolesc Health Med Ther 2014;5:191-8.

5 Kipps S, Bahu T, Ong K, et al. Current methods of transfer of young people with type 1 diabetes to adult services. Diabet Med 2002;19:649-54.

6 Scal P, Ireland M. Addressing transition to adult health care for adolescents with special health care needs. Pediatrics 2005;115:1607-12.

7 Arnett JJ. Emerging adulthood. A theory of development from the late teens through the twenties. Am Psychol 2000;55:469-80.

8 Hanberger L, Samuelsson U, Lindblad B, et al. A1C in children and adolescents with diabetes in relation to certain clinical parameters: the Swedish childhood diabetes registry SWEDIABKIDS. Diabetes Care 2008;31:927-9.

9 Lotstein DS, Seid M, Klingensmith G, et al. Transition from pediatric to adult care for youth diagnosed with type 1 diabetes in adolescence. Pediatrics 2013;131:e1062-70.

10 Miller KM, Foster NC, Beck RW, et al. Current state of type 1 diabetes treatment in the U.S.: updated data from the T1D exchange clinic registry. Diabetes Care 2015;38:971-8. 
11 Petitti DB, Klingensmith GJ, Bell RA, et al. Glycemic control in youth with diabetes: the search for diabetes in youth study. J Pediatr 2009;155:668-72.

12 Morris AD, Boyle DI, McMahon AD, et al. Adherence to insulin treatment, glycaemic control, and ketoacidosis in insulin-dependent diabetes mellitus. The DARTS/MEMO collaboration. diabetes audit and research in Tayside Scotland. medicines monitoring unit. Lancet 1997;350:1505-10.

13 Amed S, Nuernberger K, McCrea P, et al. Adherence to clinical practice guidelines in the management of children, youth, and young adults with type 1 Diabetes -A prospective population cohort study. J Pediatr 2013;163:543-8.

14 Griffin SJ. Lost to follow-up: the problem of defaulters from diabetes clinics. Diabet Med 1998;15:S14-24.

15 Sheehan AM, While AE, Coyne I. The experiences and impact of transition from child to adult healthcare services for young people with type 1 diabetes: a systematic review. Diabetic Medicine 2015;32:440-58

16 Rydall AC, Rodin GM, Olmsted MP, et al. Disordered eating behavior and microvascular complications in young women with insulindependent diabetes mellitus. N Engl J Med 1997;336:1849-54.

17 Hood KK, Huestis S, Maher A, et al. Depressive symptoms in children and adolescents with type 1 diabetes: association with diabetes-specific characteristics. Diabetes Care 2006;29:1389-91.

18 Northam EA, Matthews LK, Anderson PJ, et al. Psychiatric morbidity and health outcome in Type 1 diabetes - perspectives from a prospective longitudinal study. Diabetic Medicine 2005;22:152-7.

19 Bryden KS, Dunger DB, Mayou RA, et al. Poor prognosis of young adults with type 1 diabetes: a longitudinal study. Diabetes Care 2003;26:1052-7.

20 Bryden KS, Peveler RC, Stein A, et al. Clinical and psychological course of diabetes from adolescence to young adulthood: a longitudinal cohort study. Diabetes Care 2001;24:1536-40.

21 McPheeters M, Davis AM, Taylor JL, et al. Transition care for children with special health needs. technical brief No. 15 (prepared by the Vanderbilt university evidence-based practice center under contract No. 290-2012-00009-I). AHRQ publication No14-EHC027EF. Rockville, MD: Agency for Healthcare Research and Quality, 2014.

22 Dahlquist G, Kallen B. Mortality in childhood-onset type 1 diabetes: a population-based study. Diabetes Care 2005;28:2384-7.

23 Edge JA, Ford-Adams ME, Dunger DB. Causes of death in children with insulin dependent diabetes 1990-96. Arch Dis Child 1999;81:318-23.

24 Effect of intensive diabetes treatment on the development and progression of long-term complications in adolescents with insulindependent diabetes mellitus: diabetes control and complications trial. J Pediatr 1994;125:177-88.

25 Curtis JR, To T, Muirhead S, et al. Recent trends in hospitalization for diabetic ketoacidosis in Ontario children. Diabetes Care 2002;25:1591-6.

26 Neu A, Hofer SE, Karges B, et al. Ketoacidosis at diabetes onset is still frequent in children and adolescents: a multicenter analysis of 14,664 patients from 106 institutions. Diabetes Care 2009;32:1647-8.

27 Rewers Aet al. Predictors of acute complications in children with type 1 diabetes. JAMA 2002;287:2511-8.

28 Tieder JS, McLeod L, Keren R, et al. Variation in resource use and readmission for diabetic ketoacidosis in children's hospitals. Pediatrics 2013;132:229-36.

29 Laing SP, Swerdlow AJ, Slater SD, et al. The British diabetic association cohort study, I: all-cause mortality in patients with insulin-treated diabetes mellitus. Diabetic Medicine 1999;16:459-65.

30 Laing SP, Swerdlow AJ, Slater SD, et al. The British diabetic association cohort study, II: cause-specific mortality in patients with insulin-treated diabetes mellitus. Diabetic Medicine 1999;16:466-71.

31 Phan T-L, Hossain J, Lawless S, et al. Quarterly visits with glycated hemoglobin monitoring: the sweet spot for glycemic control in youth with type 1 diabetes. Diabetes Care 2014;37:341-5.

32 Frank M. Factors associated with non-compliance with a medical follow-up regimen after discharge from a pediatric diabetes clinic. Can J Diabetes 1996;20:13-20.

33 Nakhla M, Daneman D, To T, et al. Transition to adult care for youths with diabetes mellitus: findings from a universal health care system. Pediatrics 2009;124:e1134-41.

34 Nakhla M, Bell LE, Wafa S, et al. Improving the transition from pediatric to adult diabetes care: the pediatric care provider's perspective in Quebec, Canada. BMJ Open Diab Res Care 2017:5:e000390.

35 Michaud S, Bell L, Yale J-F, et al. The transition from pediatric to adult diabetes care: perspectives of adult care providers in Quebec. Can J Diabetes 2016;40:S46-7.
36 Egger G, Binns A, Cole M-A, et al. Shared medical appointments an adjunct for chronic disease management in Australia? Aust Fam Physician 2014;43:151-4.

37 Peters A, Laffel L, the American Diabetes Association Transitions Working Group. Diabetes care for emerging adults: recommendations for transition from pediatric to adult diabetes care systems: a position statement of the American diabetes association, with representation by the American College of osteopathic family physicians, the American Academy of pediatrics, the American association of clinical endocrinologists, the American osteopathic association, the centers for disease control and prevention, children with diabetes, the endocrine Society, the International Society for pediatric and adolescent diabetes, juvenile diabetes research Foundation international, the National diabetes education program, and the pediatric endocrine Society (formerly Lawson Wilkins pediatric endocrine Society). Diabetes Care 2011;34:2477-85.

38 Campbell F, Biggs K, Aldiss SK, et al. Transition of care for adolescents from paediatric services to adult health services. Cochrane Database Syst Rev 2016;4.

39 Wafa S, Nakhla M. Improving the transition from pediatric to adult diabetes healthcare: a literature review. Can J Diabetes 2015;39:520-8

40 Van Walleghem N, MacDonald CA, Dean HJ. Evaluation of a systems navigator model for transition from pediatric to adult care for young adults with type 1 diabetes. Diabetes Care 2008;31:1529-30.

41 Johnston $\mathrm{P}$, Bell PM, Tennet $\mathrm{H}$, et al. Audit of young people with type 1 diabetes transferring from paediatric to adult diabetic services. Pract Diab Int 2006;23:106-8.

42 Schultz AT, Smaldone A. Components of interventions that improve transitions to adult care for adolescents with type 1 diabetes. Journal of Adolescent Health 2017;60:133-46.

43 Sequeira PA, Pyatak EA, Weigensberg MJ, et al. Let's Empower and Prepare (LEAP): Evaluation of a Structured Transition Program for Young Adults With Type 1 Diabetes. Diabetes Care 2015;38:1412-9.

44 Rachas A, Lefeuvre D, Meyer L, et al. Evaluating continuity during transfer to adult care: a systematic review. Pediatrics 2016;138:e20160256.

45 Housden L, Wong ST, Dawes M. Effectiveness of group medical visits for improving diabetes care: a systematic review and metaanalysis. Can Med Assoc J 2013;185:E635-44.

46 Brazeau A-S, Wright M, Nakhla M, et al. Perception of stigma is associated with poor diabetes control among adolescents and young adults with type 1 diabetes. 20th Diabetes Canada and the Canadian Society of Endocrinology and Metabolism (CSEM) Professional Conference and Annual Meetings, 2017: S15-16.

47 Hilliard ME, Perlus JG, Clark LM, et al. Perspectives from before and after the pediatric to adult care transition: a mixed-methods study in type 1 diabetes. Diabetes Care 2014;37:346-54.

48 Ritholz MD, Wolpert H, Beste M, et al. Patient-Provider relationships across the transition from pediatric to adult diabetes care. Diabetes Educ 2014:40:40-7.

49 Wu W-C, Taveira TH, Jeffery S, et al. Costs and effectiveness of pharmacist-led group medical visits for type-2 diabetes: a multicenter randomized controlled trial. PLoS One 2018;13:e0195898.

50 Everest E, Akhtar S, Sumego M, et al. Effectiveness of shared medical appointments versus traditional clinic visits for adolescents with type 1 diabetes. Qual Manag Health Care 2016;25:181-4.

51 Rijswijk C, Zantinge E, Seesing F, et al. Shared and individual medical appointments for children and adolescents with type 1 diabetes; differences in topics discussed? Patient Educ Couns 2010;79:351-5.

52 Delamater AM, Jacobson AM, Anderson B, et al. Psychosocial therapies in diabetes: report of the psychosocial therapies Working group. Diabetes Care 2001;24:1286-92.

53 Edelman D, Gierisch JM, McDuffie JR, et al. Shared medical appointments for patients with diabetes mellitus: a systematic review. J Gen Intern Med 2015;30:99-106.

54 Quiñones AR, Richardson J, Freeman M, et al. Educational group visits for the management of chronic health conditions: a systematic review. Patient Educ Couns 2014:95:3-29.

55 Floyd BD, Block JM, Buckingham BB, et al. Stabilization of glycemic control and improved quality of life using a shared medical appointment model in adolescents with type 1 diabetes in suboptimal control. Pediatr Diabetes 2017;18:204-12.

56 Mejino A, Noordman J, van Dulmen S. Shared medical appointments for children and adolescents with type 1 diabetes: perspectives and experiences of patients, parents, and health care providers. Adolesc Health Med Ther 2012;3:75-83.

57 Raymond JK, Shea JJ, Berget C, et al. A novel approach to adolescents with type 1 diabetes: the team clinic model: Figure 1. Diabetes Spectr 2015;28:68-71. 
58 Peters A, Laffel L, American Diabetes Association Transitions Working Group. Diabetes care for emerging adults: recommendations for transition from pediatric to adult diabetes care systems: a position statement of the American diabetes association, with representation by the American College of osteopathic family physicians, the American Academy of pediatrics, the American association of clinical endocrinologists, the American osteopathic association, the centers for disease control and prevention, children with diabetes, the endocrine Society, the International Society for pediatric and adolescent diabetes, juvenile diabetes research Foundation international, the National diabetes education program, and the pediatric endocrine Society (formerly Lawson Wilkins pediatric endocrine Society). Diabetes Care 2011;34:2477-85.

59 Behan KJ, Storey NM, Lee H-K. Reporting variant hemoglobins discovered during hemoglobin $\mathrm{A} 1 \mathrm{c}$ analysis - common practices in clinical laboratories. Clinica Chimica Acta 2009;406:124-8.

60 Brazeau A-S, Nakhla M, Wright M, et al. Stigma and its association with glycemic control and hypoglycemia in adolescents and young adults with type 1 diabetes: cross-sectional study. J Med Internet Res 2018;20:e151.

61 Bandura A. Self-Efficacy: toward a unifying theory of behavioral change. Psychol Rev 1977;84:191-215.

62 Grossman HY, Brink S, Hauser ST. Self-Efficacy in adolescent girls and boys with insulin-dependent diabetes mellitus. Diabetes Care 1987;10:324-9.

63 lannotti RJ, Nansel TR, Schneider S, et al. Assessing regimen adherence of adolescents with type 1 diabetes. Diabetes Care 2006;29:2263-7.

64 lannotti RJ, Schneider S, Nansel TR, et al. Self-Efficacy, outcome expectations, and diabetes self-management in adolescents with type 1 diabetes. J Dev Behav Pediatr 2006;27:98-105.

65 Keller M, Attia R, Beltrand J, et al. Insulin regimens, diabetes knowledge, quality of life, and $\mathrm{HbA} 1 \mathrm{c}$ in children and adolescents with type 1 diabetes. Pediatr Diabetes 2017;18:340-7.

66 Martin D, Dossier C, Godot C. Questionnaire de connaissance de I'AJD pour les enfants et les adolescents ayant un diabète de type 1 . Ther Patient Educ 2016;8:1-9.
67 Martin D, Elie C, Dossier C, et al. Diabetes knowledge in adolescents with type 1 diabetes and their parents and glycemic control. Pediatr Diabetes 2017;18:559-65.

68 Moynihan M, Saewyc E, Whitehouse S, et al. Assessing readiness for transition from paediatric to adult health care: revision and psychometric evaluation of the am I on TRAC for adult care questionnaire. J Adv Nurs 2015;71:1324-35.

69 Paone M, Moynihan M, Whitehouse S ES. Am I on TRAC for adult care? youth readiness questionnaire, 2012 version. Vancouver, BC: British Columbia Children's Hospital, 2012.

70 Paone MC, Wigle M, Saewyc E. The on TRAC model for transitional care of adolescents. Prog Transp/ 2006;16:291-302.

71 Fisher L, Polonsky WH, Hessler DM, et al. Understanding the sources of diabetes distress in adults with type 1 diabetes. J Diabetes Complications 2015;29:572-7.

72 Hagger V, Hendrieckx C, Sturt J, et al. Diabetes distress among adolescents with type 1 diabetes: a systematic review. Curr Diab Rep 2016;16:9.

73 Ministère de la Santé et des Services Sociaux. Évaluation de la performance économique globale des centres hospitaliers de soins généraux et spécialisés, volet «clientèle hospitalisé» - résultats 19941995. Québec: Gouvernement du Québec, 1996.

74 Canadian Institute for Health Information. Reducing gaps in health: a focus on socio-economic status in urban Canada. Ottawa, Ontario: CIHI, 2008.

75 Pampalon R, Hamel D, Gamache P. A comparison of individual and area-based socio-economic data for monitoring social inequalities in health. Health Rep 2009;20:85-94.

76 Pampalon R, Hamel D, Gamache P. Health inequalities, deprivation, immigration and Aboriginality in Canada: a geographic perspective. Can J Public Health 2010;101:470-4.

77 Leong A, Dasgupta K, Chiasson J-L, et al. Estimating the population prevalence of diagnosed and undiagnosed diabetes. Diabetes Care 2013:36:3002-8.

78 Schmitz N, Nitka D, Gariepy G, et al. Association between neighborhood-level deprivation and disability in a community sample of people with diabetes. Diabetes Care 2009;32:1998-2004. 